\title{
Knowing When to Stop: The Brain Mechanisms of Chasing Losses
}

\author{
Daniel K. Campbell-Meiklejohn, Mark W. Woolrich, Richard E. Passingham, and Robert D. Rogers
}

Background: Continued gambling to recover previous losses ("loss-chasing") is central to pathological gambling. However, very little is known about the neural mechanisms that mediate this behavior.

Methods: We used functional magnetic resonance imaging (fMRI) to examine neural activity while healthy adult participants decided to chase losses or decided to quit gambling to prevent further losses.

Results: Chasing losses was associated with increased activity in cortical areas linked to incentive-motivation and an expectation of reward. By contrast, quitting was associated with decreased activity in these areas but increased activity in areas associated with anxiety and conflict monitoring. Activity within the anterior cingulate cortex associated with the experience of chasing and then losing predicted decisions to stop chasing losses at the next opportunity.

Conclusions: Excessive loss-chasing behavior in pathological gambling might involve a failure to appropriately balance activity within neural systems coding conflicting motivational states. Similar mechanisms might underlie the loss-of-control over appetitive behaviors in other impulse control disorders.

\section{Key Words: Decision-making, loss-chasing, motivation, pathologi- cal gambling, persistence, reward}

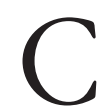
ontinued gambling to recover losses is frequently observed in both recreational gamblers (1) and in pathological gamblers (2). This behavior is known as "losschasing" (3). Loss-chasing is strongly associated with impaired control over gambling behavior and is central to and the most significant step in the development of pathological gambling (4). Left unchecked, loss-chasing can produce a dangerous spiral of accelerating involvement in gambling activities, increasing financial liabilities but diminishing resources to meet them and finally serious adverse family, social, and occupational consequences (5).

Despite the centrality of loss-chasing to pathological gambling, we know very little about its neural and neurochemical substrates. Identifying these substrates will help us to understand how the neural dysfunctions within corticolimbic circuits recently identified in samples of pathological gamblers $(6,7)$ contribute to the disorder's clinical presentation and how therapeutic interventions might promote recovery and prevent relapse.

Qualitative studies suggest that loss-chasing is driven by a mixture of motivations. On the one hand, there is the wearing anxiety associated with the gambler's already-acquired liabilities but also the persisting hope that the next gamble will be the one that clears the slate: "It's one crisis after another, and you gamble to get even .... one big hit, make that one big hit, and pay off the debts and never gamble again" (3).

On the other hand, there is the accompanying sense of dread and pessimism that yet another bad outcome will result in an even more desperate situation: "Then came the feeling .... of

From the Department of Experimental Psychology (DKC-M, REP), University of Oxford; University Department of Psychiatry (DKC-M, RDR), Warneford Hospital; and Oxford Centre for Functional Magnetic Resonance Imaging of the Brain (MWW), John Radcliffe Hospital, Oxford, United Kingdom.

Address reprint requests to Robert D. Rogers, Ph.D., University Department of Psychiatry (DCM, RDR), Warneford Hospital, Oxford, 0X3 7JX, UK; E-mail: robert.rogers@psych.ox.ac.uk.

Received December 22, 2006; revised April 23, 2007; accepted May 3, 2007. uneasiness within myself; a feeling of, probably you might call it of impending doom or disaster, that I had never had before. There was no way that I wasn't going to blow everything" (3).

In this study, we used functional magnetic resonance imaging (fMRI) in healthy adults to test the hypothesis that decisions to chase losses or to quit gambling during a series of losing gambles involve activity within neural systems that are important in the interplay between emotion and cognition, reflecting the competing motivational states underlying gamblers' loss-chasing behavior. We hypothesized that decisions to chase depend upon activity in neural pathways involved in the representation of reward expectancy, including the ventromedial prefrontal cortex (vmPFC) (8-10); whereas decisions to quit chasing losses depend upon activity in other neural circuits that are involved in visceral arousal and the anticipation of aversive consequences, including the dorsal anterior cingulate (dACC) and insula cortices (11-14).

Gamblers frequently continue to gamble for longer than originally intended to increase their winnings or recover losses sustained within a session, reflecting the increasing excitement and arousal associated with gambling behavior (15). However, loss-chasing is distinguished by the pursuit of higher-risk but higher-yield forms of gambling (such as increasing the size of bets placed) with the specific motivation of recovering money lost previously (16). Here, we modeled loss-chasing behavior by requiring healthy adult volunteers to choose repeatedly between gambling to recover a loss at the risk of doubling its size versus sustaining that loss and quitting the chase.

By investigating the neural signals associated with decisions to chase or to quit, we provide evidence that losschasing behavior-such a characteristic feature of pathological gambling-reflects a shifting balance of activity within distinct neural systems that represent its conflicting motivations. We also demonstrate that neural activity within the anterior cingulate cortex associated with deciding to chase but then losing might have a direct impact on future decisions to keep gambling. Collectively, our results provide a starting point for understanding the neural substrates of excessive loss-chasing in pathological gamblers. 


\section{Methods and Materials}

\section{Participants}

Twenty-three healthy right-handed adult volunteers (13 men; 10 women) were recruited into the study at the University of Oxford Centre for Clinical Magnetic Resonance Research. The study was approved by the National Health Service Oxfordshire Research Ethics Committee B. All participants gave full informed consent. Participants were screened to exclude any current medication, major physical or psychological illness, history of head injury, or neurological illness. The participants' mean age was $25.68 \pm 1.05$ (SEM) years; all had completed some tertiary education. Participants were paid $₫ 20$ for taking part in the study.

\section{Psychometric Questionnaires}

Participants completed the South Oaks Gambling Screen (SOGS) (17) and the Gambling Related Cognitions Scale (GRCS) (18). The GRCS has subscales for five cognitive biases associated with gambling: perceived ability to stop; predictive control; gambling expectancies; interpretive bias; and illusions of control. Participants also completed an adapted 14-item questionnaire that provided an independent assessment of participants' propensity to chase in other gambling activities (19). Participants reported infrequent involvement in real-life gambling, confined to lottery plays, poker, and occasional visits to casinos. Participants' scores on the SOGS were 0 or 1 , indicating no evidence of pathological gambling

\section{Loss-Chasing Game}

In essence, participants were required to choose between gambling to recover a loss (at the risk of doubling its size) or quitting (sustaining a certain loss). Research indicates that such dilemmas consistently induce risky choices (20). Descriptive theories of choice under uncertainty attribute this behavior to the fact that losses fall on the convex part of a psychophysical function relating monetary value to its subjective value or utility, such that the increase in utility associated with recovering previous losses is proportionately greater than the reduction in utility associated with sustaining greater losses still (21). We assumed that the neural systems involved in resolving such dilemmas would also contribute to the loss-chasing behavior observed in both recreational and pathological gamblers.

At the start of the scanning session, participants were told that they had a fictional $£ 20,000$ to play with but that the participant with the most points at the end of the study would win a real prize of 270 . On each "round" of the game, £10, £20, £40, £80, or $£ 160$ was subtracted from their game total. This amount appeared below the choices: "Quit" and "Play" (Figure 1).

At this point, participants could choose to "Quit," accepting this loss and ending the round immediately ("quit-loss" outcome), or they could choose to "Play" (i.e., chase the loss). Therefore, they could gamble on recovering an amount equal to the loss but at the risk of increasing their losses by the same amount. If the outcome of a decision to gamble was positive ("chase-win" outcome), the loss was recovered and the round ended. If the outcome was negative ("chase-loss" outcome), the loss was doubled and participants were given another chance to quit or to chase in the next choice of the round. The options for each choice-"Play" or "Quit" - appeared equally often on the left and right sides of the choice displays.

Outcome displays (Figure 1) indicated whether participants had won a gamble and that no money was lost ("chase-win"); whether they had lost a gamble and the amount lost ("chaseloss"); or the amount lost if participants chose to quit the round ("quit-loss"). At the end of each round, participants were also informed of their final losses in a "round-loss" display. This display indicated the total cumulative losses for that round, in red text if the losses were greater than 0 but in green text if 0 . Twelve rounds began with losses of $£ 10, £ 20, £ 40, £ 80$, or $£ 160$, yielding 60 rounds. If participants continued losing, losses kept doubling until they reached $₫ 640$, at which point the round ended, having incurred the maximum loss.

In summary, our participants were confronted with a series of dilemmas involving a choice between gambling to recover a loss at the risk of doubling its size or sustaining the loss and ending the chase while at the same time preserving as effectively as possible the resources that allowed play to continue. The value of this reward was provided by the context of an inter-participant competition requiring participants to retain as many points as possible.
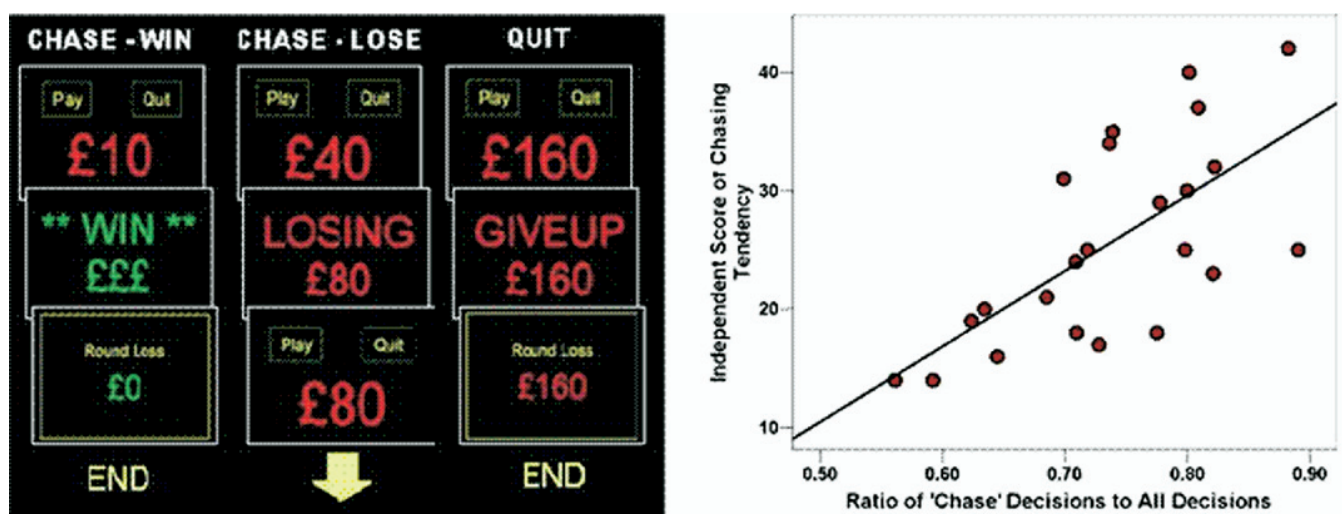

Figure 1. Display sequences for the loss-chasing game. The game consisted of 60 rounds of loss-chasing, each with a minimum of one and a maximum of six choices. At the beginning of each round, a loss was imposed and a decision made either to play (gamble further) or quit (to accept the loss) and end the round. This was followed by presentation of the outcome of that choice. Consecutive losses and decisions occurred until a maximum round loss of $£ 640$ was incurred participants won a gamble and cleared their losses ("chase-win" outcome), or participants chose to quit ("quit-loss" outcome) at which point the round ended. At the end of each round, participants were informed of their final round losses ("round-loss" outcomes). The number of decisions to chase losses in the game showed a positive association $(r=.67, p<.001)$ with a psychometric measure of chasing behavior in other gambling situations that was adapted for the current experiment (19). This questionnaire consisted of 14 items with Likkert-scale ratings between "1/Occasionally" and " $5 /$ Almost Always" and of statements such as "After losing heavily: I felt an urge to continue betting " and "After losing heavily: I thought I would like to increase the size of my bets." 
Finally, the outcomes of the loss-chasing game were structured such that $83 \%$ of rounds would eventually return all losses and $17 \%$ would result in the maximum loss of $£ 640$ if participants decided to play and not to quit on every choice in the game. Chase-win outcomes were positioned randomly within each round such that a winning outcome would occur equally often after any number of (between 0 and 5) consecutive losses. To discourage participants from adopting conservative strategies by which they quit early to preserve as much of their play money as possible, no information was provided about their cumulative game total of play money during the game. Participants were also informed that they would not achieve the best possible score by exclusively playing or quitting.

\section{Control Task}

We also included a separate control task in which no decision had to be made before a response, so as to control for the overall visual and motor demands of our loss-chasing game. This control task served as a common comparison for decisions to chase losses and decisions to quit. Control displays ("choice-control," "outcome-control," "round-loss-control" displays) were identical to the corresponding game displays (and durations) in all respects except that alphanumeric characters were replaced with the symbol "\#". In choice-control displays, the word "Press" appeared in one of the two yellow boxes (randomly allocated) on the left or right of the displays. If the word appeared on the left, participants were required to make a left button-press; if the word appeared on the right, participants were required to make a right button-press. After this, participants attended to the subsequent control-outcome displays. One-third of the control rounds proceeded to a round-loss-control feedback display. Participants performed one round of the control task (two or three responses) every 10 rounds of the loss-chasing game.

Details of the stimulus presentation, the trial structures of the loss-chasing game and control task (including the separation of overlapping hemodynamic responses evoked by consecutive events), and participants' training are provided in Supplement 1.

\section{Behavioral Data: Statistical Analysis}

The dependent measures of our loss-chasing game were the proportion of decisions to chase out of all of the decisions made during the game and the mean deliberation time for these decisions. Differences among the mean deliberation times for decisions to chase, decisions to quit, and participants' responses on the control task were tested with paired, 2-tailed $t$ tests. All correlations between proportion of decisions to chase and psychometric measures were assessed with 2-tailed Pearson correlations.

\section{Functional Imaging: Acquisition}

Participants were scanned at 3 Teslas with a Siemens MAGNETUM Trio scanner (Siemens Medical Solutions, Erlangen, Germany). A T2-weighted echo planar image (EPI) sequence was optimized for signal contrast in VmPFC, with a tilt angle of $30^{\circ}$ and a preparation pulse in the slice selection direction to compensate for through-plane susceptibility gradients (22). A T1-weighted anatomical dataset was acquired for coregistration with the EPI data. Further details are provided in Supplement 1.

\section{Functional Imaging Data: Preprocessing and Modeling}

Image preprocessing and analysis was carried out with FEAT (FMRI Expert Analysis Tool) version 5.43, which is part of FMRIB's (Oxford Centre for Functional Magnetic Resonance Imaging of the Brain) software library (http://www.fmrib.ox. ac.uk/fsl). Preprocessing of the EPI data sequences consisted of removal of non-brain matter, high-pass filtering, motion realignment, slice-time correction, smoothing with Gaussian filter (fullwidth half-maximum of $5 \mathrm{~mm}$ ) and compensation for geometric distortion and signal loss (23).

Standard general linear models (GLM) were used for individual EPI sequences, providing contrasts for group effects analyzed at the higher level. Single-participant GLM results were estimated (24) and transformed, after spatial normalization, into standard (Montreal Neurological Institute [MNI]152) space (25). Group analyses were carried out with FMRIB's Local Analysis of Mixed Effects (24). The GLM analyses calculated the mean effect across all $Z$ (Gaussianized T/F) statistical images. The $Z$ (Gaussianised $\mathrm{T} / \mathrm{F}$ ) statistical images were thresholded with clusters determined by $Z>2.3$ and a (whole-brain corrected) cluster significance threshold of $p<.05$ (26-28).

Statistical tests included contrasts between chase, quit, and control task regressors and between regressors for each outcome (chase-wins, chase-losses, and quit-losses). We also modeled subtypes of losing outcome (those before decisions to chase again and those before decisions to quit) and tested the effects of the Interpretive Bias subscale of GRCS as a negative covariate. Details of the image analysis are provided in Supplement 1.

\section{Results}

\section{Validity of the Loss-Chasing Game}

On average, participants chose to chase the loss on $73 \pm 2 \%$ of all decisions and the mean number of chases/round was 2.07 trials ( $\pm .07 ; \min 1.5, \max 2.88$ ). Therefore we found that, as predicted, participants were motivated to gamble to recover points lost at the start of each round of the game or through the bad outcomes of earlier decisions to chase. The proportion of decisions to chase showed a strong association with the total score on the 14-item psychometric assessment of participants' propensity to chase in other gambling activities (19) $(r=.67, p<$ .001 ; Figure 1). Therefore, our loss-chasing game shows some external validity for chasing behavior in other forms of gambling.

Participants' deliberation times when deciding to quit chasing losses during our loss-chasing game (2236 $\pm 144 \mathrm{msec})$ were significantly longer than their deliberation times when deciding to chase losses $(1827 \pm 117 \mathrm{msec} ; p<.001)$. Both of these decisions took significantly longer than the time taken to respond to the control task $(1027 \pm 65.27 \mathrm{msec} ; p$ values $<.0001)$.

\section{Comparisons of Brain Activity Related to Decisions to Chase and Decisions to Quit}

We first compared the blood oxygen level dependent (BOLD) amplitudes that were associated with decisions to chase with the BOLD amplitudes that were associated with decisions to quit (Table 1). Decisions to chase were associated with increased neural activity within the vmPFC along the gyrus rectus on the left and within the subgenual cingulate cortex bilaterally (sgACC) (area 25) (Figure 2).

By contrast, comparison of decisions to quit chasing with decisions to chase the loss revealed a quite different pattern of BOLD signals (Table 1). There was increased activity within the dACC, the ventral striatum, and anterior insula cortices (Figure 2). The activity in the dACC included not only the anterior cingulate proper (area 24) but also the paracingulate cortex (area 32). Table 1 indicates that there were also substantial signal increases along the middle frontal gyrus, in the posterior cingulate cortex, and in bilateral parietal cortices. 
Table 1. Differences in BOLD Signal Associated With Decisions to Chase Losses Compared With Decisions to Quit in the Loss-Chasing Game

\begin{tabular}{|c|c|c|c|c|c|c|}
\hline \multirow[b]{2}{*}{ Location } & \multirow[b]{2}{*}{ Side } & \multicolumn{3}{|c|}{ Coordinates $(\mathrm{mm})$} & \multirow[b]{2}{*}{ Z Score } & \multirow{2}{*}{$\begin{array}{c}\text { Cluster Size } \\
\text { (Voxels) }\end{array}$} \\
\hline & & $x$ & y & z & & \\
\hline \multicolumn{7}{|c|}{$\begin{array}{l}\text { Activity greater during decisions to chase losses compared with activity during } \\
\text { decisions to quit }\end{array}$} \\
\hline ventromedial prefrontal cortex, gyrus rectus (anterior peak) & L & -6 & 50 & -16 & 4.15 & 574 \\
\hline ventromedial prefrontal cortex, gryus rectus (posterior peak) & L & -6 & 38 & -18 & 3.34 & \\
\hline subgenual anterior cingulate cortex, cingulate gyrus & $\mathrm{L}^{a}$ & -2 & 6 & -12 & 3.13 & \\
\hline parietal cortex, angular gyrus & $\mathrm{L}$ & -54 & -70 & 26 & 3.82 & 421 \\
\hline \multicolumn{7}{|c|}{$\begin{array}{l}\text { Activity greater during decisions to quit compared with activity during decisions } \\
\text { to chase losses }\end{array}$} \\
\hline dorsal anterior cingulate cortex, cingulate gyrus & $\mathrm{L}^{a}$ & -4 & 22 & 38 & 5.47 & 16,979 \\
\hline anterior insula, short insular gyri & $\mathrm{R}$ & 36 & 18 & 0 & 5.42 & \\
\hline prefrontal cortex, middle frontal gyrus & $\mathrm{R}$ & 38 & 30 & 36 & 4.47 & \\
\hline prefrontal cortex, middle frontal gyrus & $\mathrm{R}$ & 40 & 8 & 52 & 4.46 & \\
\hline insula, anterior, short insular gyri & $\mathrm{L}$ & -32 & 20 & 2 & 4.69 & \\
\hline mid posterior cingulate cortex, cingulate gyrus & $-^{a}$ & 0 & -30 & 26 & 4.55 & \\
\hline striatum, caudate nucleus/putamen & $\mathrm{L}$ & -18 & 18 & -4 & 3.65 & \\
\hline striatum, caudate nucleus/putamen & $\mathrm{R}$ & 18 & 18 & -6 & 3.62 & \\
\hline parietal cortex, inferior parietal gyrus & $\mathrm{L}$ & 44 & -52 & 52 & 5.2 & 23,938 \\
\hline parietal cortex, inferior parietal gyrus & $\mathrm{L}$ & -42 & -44 & 48 & 4.83 & \\
\hline occipital cortex, cuneus & $\mathrm{R}^{a}$ & 8 & -74 & 6 & 4.57 & \\
\hline parietal cortex, precuneus & $\mathrm{R}^{a}$ & 4 & -74 & 44 & 4.47 & \\
\hline
\end{tabular}

Coordinates $(\mathrm{mm})$ are provided for peak voxels of clustered activity and are standardized to Montreal Neurological Institute (MNI) space. BOLD, blood oxygen level dependent.

${ }^{a}$ Bilateral cluster activation.

These data suggest that loss-chasing is mediated by activity within neural systems that represent an expectation of positive outcomes $(8,10)$, whereas decisions to stop chasing are mediated by activity within systems associated with negative affect $(12,14)$. In light of these findings, we sought to investigate whether deciding to chase losses or deciding to quit depended upon a balance of activity within dissociable neural networks, by comparing the BOLD signals associated with each of these choices against the common control condition.

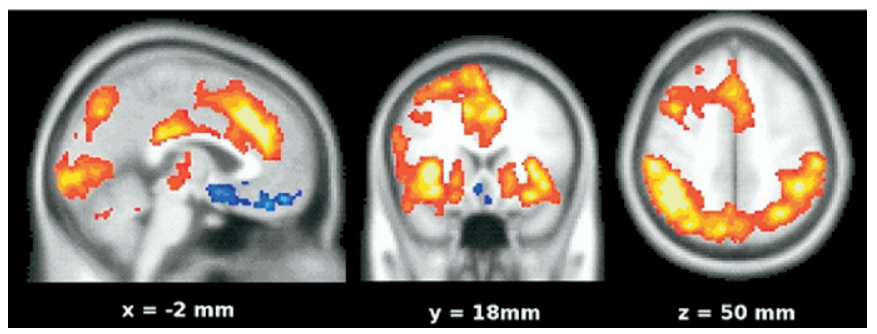

Figure 2. Anatomical dissociation between decisions to chase losses and decisions to quit. Decisions to chase losses (double or nothing) compared with decisions to quit (accepting the immediate loss) were associated with significant increases in blood oxygen level dependent (BOLD) amplitudes illustrated in dark blue $(Z$ score $=2.3)$ to light blue ( $\max Z$ score), including areas of the ventromedial prefrontal cortex (vmPFC) (MNI coordinates [mm]: $-6,50,-16)$ and the subgenual anterior cingulate cortex (sgACC) $(-2,6$, -12 ). By contrast, decisions to quit compared with decisions to chase losses were associated with increased BOLD amplitudes illustrated in red $(Z$ score $=$ 2.3) to yellow ( $\max Z$ score), encompassing areas of bilateral anterior insula $(36,18,0)$, dorsal anterior cingulate $(-4,22,38)$, posterior cingulate $(0,-30$, 26), parietal cortices, and ventral striatum $(-18 / 18,18,-4)$. Group data (thresholded with cluster correction at $p<.05$ ) is rendered onto a standard MNI152 brain image.

\section{Comparisons of Brain Activity When Deciding to Chase or Quit and the Control Condition}

In comparison with the control condition, decisions to quit were associated with increased activity within the dACC, left anterior insula, posterior cingulate, and parietal cortices (Table 2; Figure 3) but decreased activity within the vmPFC and sgACC (Figure 3). Complementing this clear dissociation, decisions to chase were not associated with any increase in activity in comparison with the control condition but were associated with a decrease of activity in the AACC, right anterior insula, and inferior frontal gyrus (Table 2). These reductions correspond to areas isolated in the direct comparisons between decisions to chase and decisions to quit.

These signal changes could not have arisen artefactually through differences in the mean or the variability of the monetary losses accumulated before decisions to chase compared with decisions to quit, because we found exactly the same distribution of BOLD signals when the aforementioned comparisons were repeated with subsets of choices in which these factors were precisely balanced (Supplement 1). We also replicated the aforementioned results when we compared sub-sets of decisions to chase and decisions to quit that were matched for the mean number (and variability) of preceding losses (Supplement 1). Additional analyses also demonstrated that only signal changes within the cingulate cortex showed any direct association with deliberation times for decisions to quit (see Supplement 1).

Finally, we also investigated the influence of individual variability in the tendency to reinterpret gambling losses in such a way as to promote continued play. Such cognitions involve attributing "successes to one's own skill and failures to others' influences or luck or recalling wins more easily than losses and thus expecting to win at games that they have lost previously" and are reflected in participants' scores on the Interpretive Bias subscale of the GRCS (18). We entered participants' scores on the 
Table 2. Differences in BOLD Signal Associated With Decisions to Chase, Decisions to Quit, and Responses in the Control Condition

\begin{tabular}{|c|c|c|c|c|c|c|}
\hline \multirow[b]{2}{*}{ Location } & \multirow[b]{2}{*}{ Side } & \multicolumn{3}{|c|}{ Coordinates $(\mathrm{mm})$} & \multirow[b]{2}{*}{ Z Score } & \multirow{2}{*}{$\begin{array}{l}\text { Cluster Size } \\
\text { (Voxels) }\end{array}$} \\
\hline & & $x$ & y & z & & \\
\hline \multicolumn{7}{|c|}{$\begin{array}{l}\text { Activity greater during decisions to quit compared with responses in the } \\
\text { control condition }\end{array}$} \\
\hline dorsal anterior cingulate cortex, cingulate gyrus & $L^{a}$ & -2 & 26 & 36 & 4.91 & 2356 \\
\hline parietal cortex, inferior parietal gyrus & $\mathrm{L}$ & -42 & -48 & 52 & 3.69 & 2056 \\
\hline parietal cortex, inferior parietal gyrus & $\mathrm{R}$ & 42 & -52 & 52 & 4.15 & 2034 \\
\hline thalamus & $L^{a}$ & -8 & -18 & 6 & 4.31 & 1120 \\
\hline anterior insula, short insular gyri & L & -30 & 20 & 4 & 3.73 & 413 \\
\hline mid posterior cingulate cortex, cingulate gyrus & $-^{a}$ & 0 & -30 & 26 & 3.44 & 409 \\
\hline \multicolumn{7}{|c|}{ Activity greater during control condition compared with decisions to quit } \\
\hline occipital cortex, cuneus & $L^{a}$ & -6 & -88 & -8 & 4.91 & 5841 \\
\hline ventromedial prefrontal cortex, gyrus rectus & L & -6 & 50 & -16 & 3.44 & 501 \\
\hline subgenual anterior cingulate cortex, cingulate gyrus & $\mathrm{R}^{a}$ & 4 & 20 & -10 & 3.06 & \\
\hline \multicolumn{7}{|c|}{$\begin{array}{l}\text { Activity greater during control condition compared with decisions to } \\
\text { chase losses }\end{array}$} \\
\hline occipital cortex, cuneus & $\mathrm{R}^{a}$ & 4 & -84 & -14 & 6.76 & 34,242 \\
\hline anterior insula, short insular gyri & $\mathrm{R}$ & 36 & 18 & 2 & 3.93 & \\
\hline prefrontal cortex, inferior frontal gyrus & $\mathrm{R}$ & 52 & 40 & 4 & 3.83 & \\
\hline dorsal anterior cingulate cortex, cingulate gyrus & $\mathrm{R}$ & 12 & 18 & 42 & 3.06 & \\
\hline mid anterior cingulate cortex, cingulate gyrus & $\mathrm{R}$ & 10 & 50 & 14 & 2.96 & 414 \\
\hline
\end{tabular}

Coordinates $(\mathrm{mm})$ are provided for peak voxels of clustered activity and are standardized to Montreal Neurological Institute (MNI) space. BOLD, blood oxygen level dependent.

${ }^{a}$ Bilateral cluster activation.

Interpretive Bias subscale as a negative covariate of interest in the comparison of decisions to quit against the visuo-motor control task (Supplement 1). Participants with high interpretative bias showed reduced activity in precisely those areas found to be active when deciding to quit the chase: namely, the dACC and paracingulate region, the striatum on the left, the posterior cingulate, and parietal cortices (Supplement 1).

\section{Using Bad Outcomes to Stop Chasing}

There was evidence that the positive and negative outcomes of decisions to chase were associated with increased signal within neural systems associated with reinforcement learning. Comparing the good outcomes of decisions to chase ("chase-win outcomes") with the bad outcomes ("chase-loss outcomes") revealed increased activity within the medial prefrontal cortex, striatum, and posterior cingulate cortex (Supplement 1). Both good and bad outcomes were associated with increased activity within bilateral ventral striatum and putamen compared with processing the results of having quit ("quit-loss outcomes").

Finally, we also examined whether the neural processes activated by losses arising out of decisions to chase affected subsequent decisions to chase again or decisions to quit. Comparison of "chase-loss outcomes" followed by a decision to quit and "chase-loss outcomes" followed by decision to chase revealed significantly greater activity within the dACC (Figure 4). This activity was located within the same region of the dACC as that previously observed to be more active during decisions to quit compared with decisions to chase (Table 1).

\section{Discussion}

We started by demonstrating that our loss-chasing game shows some validity as a measure of young healthy adults" tendency to gamble to recover losses. Decisions to gamble to recover losses correlated well with a psychometric measure of the propensity to chase in other gambling situations. This result allowed us to look at neural activity associated either with deciding to chase losses or deciding to quit within a series of losing gambles. We found an impressive dissociation between the cortical and sub-cortical systems involved in these decisions. In the following text, we discuss our findings and the clues they provide about the neural substrates of the excessive loss-chasing behavior observed in pathological gamblers.

Gamblers' accounts of their experiences indicate that losschasing is sustained by the belief that winning outcomes are imminent (29). Our imaging findings provide important evidence to support this claim. Compared with deciding to quit, deciding to chase involved activity in two areas: the vmPFC and sgACC. This is consistent with our hypothesis that loss-chasing reflects neural activity associated with an expectancy of positive outcomes (8). It is also consistent with findings that the vmPFC represents the output of reinforcement processes determining the value of goal-directed actions (30).

The sgACC has been similarly implicated in aspects of strong appetitive states such as hunger (31), and there is evidence that, in healthy volunteers, this region plays a role in representing positive as well as negative emotions $(32,33)$. Loss-chasing frequently involves a strong appetitive component, manifested in uncontrollable urges to continue gambling or increase the size of bets placed. The experience of urges or cravings after infusions of cocaine in cocaine-dependent individuals is associated with increased activity within the sgACC (34). Thus, our findings suggest that decisions to chase are mediated by activity in systems that code positive incentive-value and powerful appetitive states and that dysfunction in these circuits mediates the excessive urge to chase reported by pathological gamblers $(3,35)$.

We found a contrasting pattern of results when our participants decided to quit gambling. Decisions to quit were associated with activity within the dACC, striatum, and bilateral anterior insula as well as the posterior cingulate and parietal regions. There is frequently co-activation of the dACC and the anterior insula $(13,36)$, reflecting strong connections between these cor- 

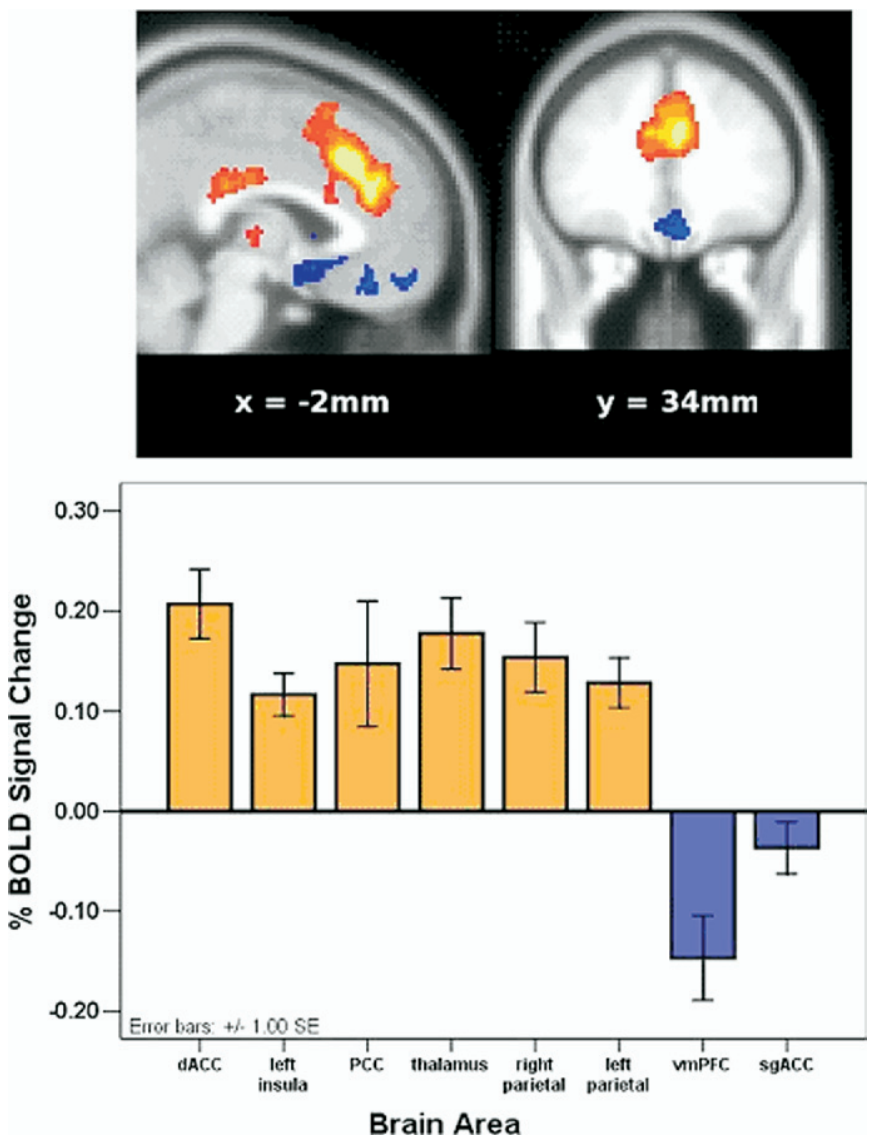

Figure 3. Activity associated with decisions to quit versus responses in the control condition. Decisions to quit chasing losses demonstrated an altered balance of activity between two systems that are associated with chasing behavior. Areas in which there was increased activity during decisions to quit compared with control responses are shown in red $(Z$ score $=2.3)$ to yellow ( $\max Z$ score). These include the dorsal anterior cingulate cortex (dACC) $(-2,26,36)$, left anterior insula $(-30,20,4)$, the posterior cingulate gyrus $(0,-30,26)$, thalamus on the left $(-8,-18,6)$, and bilateral inferior parietal gyrus $(-42,-48,52$ and $42,-52,52)$. Areas in which there was reduced activity during decisions to quit compared with responses in the control condition are shown in blue. These include $\operatorname{vmPFC}(-6,50,-16)$ and $\operatorname{sgACC}(4,20,-10)$. Group data (thresholded with cluster correction at $p<$ .05 ) is rendered onto a standard MNI152 brain image. Histograms for peak voxel \% BOLD signal change between decisions to quit and control responses. Areas in which there was increased activity for decisions to quit are shown in orange; areas in which there was decreased activity for decisions to quit are shown in blue. $\mathrm{PCC}=$ posterior cingulate cortex; other abbreviations as in Figure 2.

tical areas $(37,38)$, whereas both the dACC and anterior insula cortices send strong efferent projections to the striatum (39). Animal and human studies have shown that activity within the more posterior cingulate and parietal cortex is sensitive to the uncertainty of rewards linked to candidate actions (40) and to be involved in integrating uncertainty and reward information to determine the value of candidate actions (41).

Activity within the dACC and insula areas might represent future bad outcomes that motivate decisions to quit. The anterior insula plays a significant role in representing negative states such as pain and disgust $(11,14)$ and the anticipation of aversive stimuli (12,13). Similarly, the dACC (area 24) might be involved in anticipating bad outcomes through its role in pain processing (12). Insula activity has been linked to trait harm avoidance while making decisions (42) and to precede risk-free choices in a financial investment task (43). There is also evidence that the anterior insula represents the visceral sensations that provide a substrate for the subjective awareness of emotionally potent information (36), raising the possibility that the visceral representations associated with decisions to quit gambling are weakened in individuals who exhibit excessive loss-chasing as part of their pathological gambling.

Loss-chasing can also involve prolonged cognitive activity as gamblers weigh the pros and cons of continuing to gamble or stop (44). In our study, participants might have experienced particular conflict for those choices resulting in decisions to quit, as evidenced by significantly lengthened deliberation times. The dACC and anterior insula might also have been involved in resolving this conflict in favor of decisions to quit. Activity within the dACC has been reported in situations in which participants monitor cognitive states involving conflict or competition between activated responses $(45,46)$ or in situations requiring participants to switch between actions associated with affectively significant outcomes $(43,47)$. Activity in the anterior insula has also been shown to be increased specifically under condition of ambiguity—such as here in our loss-chasing game-in which the probabilities of good and bad outcomes are not clearly defined (48). Thus, the increased time needed to decide to quit might reflect the interdiction of processing within neural systems implicated in the monitoring of cognitive and response conflicts, the appraisal of risk and the value of candidate actions, and the subjective experience of emotional arousal.

Evidence that altered activity within neural circuits supporting these processes might predispose vulnerable individuals to excessive loss-chasing behavior is provided by our examination of individual differences in the way participants thought about winning and losing. We found that participants who tended to interpret outcomes in a way that encourages continued play were the same participants who showed reduced activity in precisely those areas associated with decisions to quit, namely, the dACC, the striatum, middle frontal gyrus, and the posterior cingulate and parietal cortices. This suggests that pathological gamblers or individuals whose thinking about gambling might make them prone to loss-chasing show altered patterns of activity in those neural systems that support decisions to quit.

The previous discussion suggests that decisions to chase

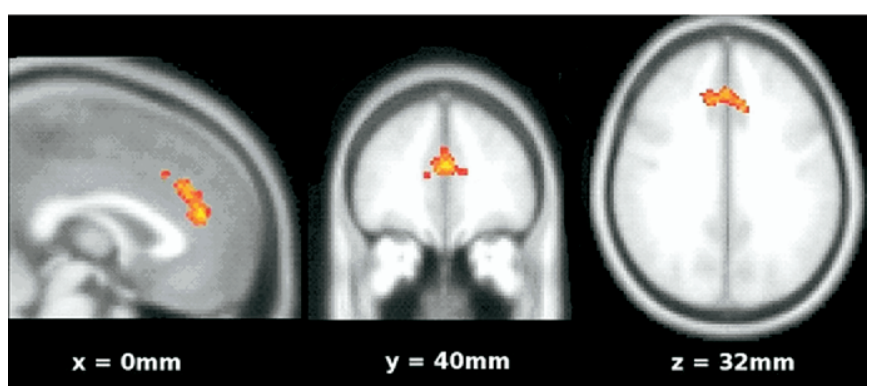

Figure 4. Using bad outcomes to stop chasing losses. Comparison of BOLD amplitudes associated with losses followed by decisions to quit on the next opportunity and BOLD amplitudes associated with losses followed by decisions to chase the loss again. Dorsal anterior cingulate cortex activity (MNI coordinates $[\mathrm{mm}] 2,34,28 ; Z$ score $=3.23$ ) is greater during a loss outcome followed by a decision to quit the chase. Thresholded image shown in red $(Z$ score $=2.3$ ) to yellow $(Z$ score $=3.23$ ). Position of view in MNI coordinates. Analysis performed as described in materials and methods. Group data (thresholded with cluster correction at $p<.05$ ) is rendered onto a standard MNI152 brain image. Abbreviations as in Figure 2. 
losses or quit depend upon a balance between distinct neural systems that represent the conflicting motivations (3). Direct evidence for balance between those systems coding participants' reward expectancy and those coding negative emotional states was provided by comparisons of chasing and quitting with the control condition in which no decision was required. When the participants decided to quit gambling, there was reduced activity in areas activated during chasing (i.e., the vmPFC and sgACC). When participants decided to chase losses, there was reduced activity in areas activated during quitting (i.e., the dACC and anterior insula). It is unclear from our results whether, in the context of loss-chasing behavior, activity within the dACC and anterior insula cortex is dynamically related to activity within the vmPFC and sgACC. One possibility is that the decrease in activity observed in the vmPFC associated with decisions to quit might also reflect the need to attenuate emotional signals-perhaps those associated with expected reward-as cognitive demands increase (49). Research will need to establish whether these two systems participate in a reciprocal or "seesaw" relationship and whether over- or under-activity in either system alone can promote loss-chasing behavior.

Winning back money lost through previous gambles or losing still more money after decisions to chase were both associated with increased activity within the medial prefrontal cortex and ventral striatum (extending into the putamen) compared with processing the known losses associated with decisions to quit. Activity within the ventral striatum has been linked to reward processing and, in particular, a role in registering deviations from expected reward (50). The ventral striatum also plays a role in resolving choices between competing actions on the basis of their values (51). Brain-imaging studies suggest that pathological gamblers show reduced activity within the ventral striatum (and the vmPFC) both while viewing gambling-related pictures (6) and while playing a guessing game for monetary reward (7), suggesting that the disorder involves hypoactivity within mesolimbic reward pathways (7). Our finding that processing both the good and bad outcomes of decisions to chase (and deciding to quit gambling itself) is associated with increased activity within the ventral striatum and the vmPFC suggests that dysfunction in mesolimbic reinforcement pathways can contribute to excessive loss-chasing behavior in pathological gamblers.

We have also demonstrated how differences in the processing of losses arising out of decisions to keep gambling help promote or inhibit subsequent chasing behavior. Specifically, we compared signal associated with losses followed by decisions to quit on the subsequent choice with signal associated with losses followed by decisions to chase again. This comparison revealed that losses followed by decisions to quit involved increased neural activity within the dACC compared with losses followed by decisions to chase. This same cingulate area was also observed to be activated when simply deciding to quit in a run of losing gambles. Several sources of evidence implicate the dACC in learning about the value of actions $(47,52)$ and in integrating the risk of an action with its value in order to optimize subsequent decision-making (53). This important finding suggests that variation in the engagement of the dACC when processing the bad outcomes of decisions to chase might account for how one such gamble can lead to another, promoting loss-chasing behavior.

Finally, our findings raise the question of how neural activity that supports loss-chasing behavior within a single gambling session contributes to gambling behavior that persists acrosssessions (16). Problem gamblers often resume gambling, explicitly with the motivation of recovering losses incurred during previous sessions $(4,29)$. However, there is evidence that chasing "within a session is a developmental forerunner of returning later to chase" (44). Follow-up research might examine whether the pattern of neural activity supporting loss-chasing behavior is more readily re-instantiated in vulnerable individuals, perhaps reflecting an easily accessible belief that continued gambling will clear the slate before the available resources run out: "He sees himself getting in deeper and deeper: yet if he quits now, all this is irretrievably lost. The only way to get it back is to keep playing ..." (3).

This research was funded by a Medical Research Council studentship to DC-M and by an independent award from the Biotechnology and Biological Sciences Research Council (United Kingdom) to RDR. We report no biomedical financial interests or potential conflicts of interest.

We would like to thank Dr. Peter Talbot for helpful suggestions about an earlier draft and Prof. Peter Jezzard and Dr. Mark Jenkinson for help optimizing the fMRI sequences and remedying signal distortion.

Supplementary material cited in this article is available online.

1. Dickerson M, Hinchy J, Fabre J (1987): Chasing, arousal and sensation seeking in off-course gamblers. Br J Addict 82:673-680.

2. American Psychiatric Association (2000): Diagnostic and Statistical Manual of Mental Disorders, Fourth Edition, Text Revision. Washington DC: American Psychiatric Publishing.

3. Lesieur HR (1984): The Chase: Career of the Compulsive Gambler. Cambridge, Massachusetts: Schenkman.

4. Lesieur HR (1979): The compulsive gambler's spiral of options and involvement. Psychiatry 42:79-87.

5. Corless T, Dickerson M (1989): Gamblers' self-perceptions of the determinants of impaired control. Br J Addict 84:1527-1537.

6. Potenza MN, Steinberg MA, Skudlarski P, Fulbright RK, Lacadie CM, Wilber MK, et al. (2003): Gambling urges in pathological gambling: A functional magnetic resonance imaging study. Arch Gen Psychiatry 60: 828-836.

7. Reuter J, Raedler T, Rose M, Hand I, Glascher J, Buchel C (2005): Pathological gambling is linked to reduced activation of the mesolimbic reward system. Nat Neurosci 8:147-148.

8. Knutson B, Fong GW, Bennett SM, Adams CM, Hommer D (2003): A region of mesial prefrontal cortex tracks monetarily rewarding outcomes: Characterization with rapid event-related fMRI. Neuroimage 18: 263-272.

9. Schoenbaum G, Chiba AA, Gallagher M (1998): Orbitofrontal cortex and basolateral amygdala encode expected outcomes during learning. Nat Neurosci 1:155-159.

10. Knutson B, Taylor J, Kaufman M, Peterson R, Glover G (2005): Distributed neural representation of expected value. J Neurosci 25:4806-4812.

11. Singer T, Seymour B, O'Doherty J, Kaube H, Dolan RJ, Frith CD (2004): Empathy for pain involves the affective but not sensory components of pain. Science 303:1157-1162.

12. Ploghaus A, Tracey I, Gati JS, Clare S, Menon RS, Matthews PM, et al. (1999): Dissociating pain from its anticipation in the human brain. Science 284:1979-1981.

13. Critchley HD, Wiens S, Rotshtein P, Ohman A, Dolan RJ (2004): Neural systems supporting interoceptive awareness. Nat Neurosci 7:189-195.

14. Wicker B, Keysers C, Plailly J, Royet JP, Gallese V, Rizzolatti G (2003): Both of us disgusted in My insula: The common neural basis of seeing and feeling disgust. Neuron 40:655-664.

15. Nower L, Derevensky JL, Gupta R (2004): The relationship of impulsivity, sensation seeking, coping, and substance use in youth gamblers. Psychol Addict Behav 18:49-55.

16. Walker MB (1992): The Psychology of Gambling: Oxford, Pergamon Press.

17. Lesieur HR, Blume SB (1987): The South Oaks Gambling Screen (SOGS): A new instrument for the identification of pathological gamblers. $A m J$ Psychiatry 144:1184-1188. 
18. Raylu N, Oei TP (2004): The Gambling Related Cognitions Scale (GRCS): Development, confirmatory factor validation and psychometric properties. Addiction 99:757-769.

19. O'Connor J, Dickerson M (2003): Definition and measurement of chasing in off-course betting and gaming machine play. J Gambl Stud 19: 359-386.

20. Shafir E, Tversky A (1995): Decision making. In: Smith EE, Oscherson DN, editors. Thinking. Cambridge, Massachusetts: MIT Press, 77-100.

21. Khaneman D, Tversky A (2000): Choices Values and Frames. Cambridge, United Kingdom: Cambridge University Press.

22. Deichmann R, Gottfried JA, Hutton C, Turner R (2003): Optimized EPI for fMRI studies of the orbitofrontal cortex. Neuroimage 19:430-441.

23. Jenkinson M (2003): Fast, automated, N-dimensional phase-unwrapping algorithm. Magn Reson Med 49:193-197.

24. Woolrich MW, Behrens TEJ, Beckmann CF, Jenkinson M, Smith SM (2004): Multilevel linear modelling for FMRI group analysis using Bayesian inference. Neurolmage 21:1732-1747.

25. Jenkinson M, Bannister P, Brady M, Smith S (2002): Improved optimization for the robust and accurate linear registration and motion correction of brain images. Neurolmage 17:825-841.

26. Forman SD, Cohen JD, Fitzgerald M, Eddy WF, Mintun MA, Noll DC (1995): Improved assessment of significant activation in functional magnetic resonance imaging (fMRI): Use of a cluster-size threshold. Magn Reson Med 33:636-647.

27. Friston KJ, Worsley KJ, Erackowiak RSJ, Mazziotta JC, Evans AC (1994): Assessing the significance of focal activations using their spatial extent. Hum Brain Mapp 1:214-220.

28. Worsley KJ, Evans AC, Marrett S, Neelin P (1992): A three-dimensional statistical analysis for CBF activation studies in human brain. $J$ Cereb Blood Flow Metab 12:900-918.

29. Ladouceur R, Walker M (1996): A cognitive perspective on gambling. In: Salkovskis PM, editor. Trends in Cognitive and Behavioral Therapies. New York: Wiley.

30. Hampton AN, Bossaerts P, O'Doherty JP (2006): The role of the ventromedial prefrontal cortex in abstract state-based inference during decision making in humans. J Neurosci 26:8360-8367.

31. Tataranni PA, Gautier JF, Chen K, Uecker A, Bandy D, Salbe AD, et al. (1999): Neuroanatomical correlates of hunger and satiation in humans using positron emission tomography. Proc Natl Acad Sci U S A 96:4569-4574.

32. Damasio AR, Grabowski TJ, Bechara A, Damasio H, Ponto LL, Parvizi J, et al. (2000): Subcortical and cortical brain activity during the feeling of self-generated emotions. Nat Neurosci 3:1049-1056.

33. Lane RD, Reiman EM, Ahern GL, Schwartz GE, Davidson RJ (1997): Neuroanatomical correlates of happiness, sadness, and disgust. Am J Psychiatry 154:926-933.

34. Breiter HC, Gollub RL, Weisskoff RM, Kennedy DN, Makris N, Berke JD, et al. (1997): Acute effects of cocaine on human brain activity and emotion. Neuron 19:591-611.

35. Dickerson MG (1991): Internal and external determinants of persistent gambling. In: Heather N, Miller WM, Greeley J, editors. Self-Control and Addictive Behaviours. Sydney: Maxwell Macmillan, 317-338.
36. Craig AD (2002): How do you feel? Interoception: The sense of the physiological condition of the body. Nat Rev Neurosci 3:655-666.

37. Ongur D, Price JL (2000): The organization of networks within the orbital and medial prefrontal cortex of rats, monkeys and humans. Cereb Cortex 10:206-219.

38. Mesulam MM, Mufson EJ (1982): Insula of the old world monkey. III: Efferent cortical output and comments on function. J Comp Neurol 212: $38-52$.

39. Chikama M, McFarland NR, Amaral DG, Haber SN (1997): Insular cortical projections to functional regions of the striatum correlate with cortical cytoarchitectonic organization in the primate. J Neurosci 17:9686-9705.

40. McCoy AN, Platt ML (2005): Risk-sensitive neurons in macaque posterior cingulate cortex. Nat Neurosci 8:1220-1227.

41. Platt ML, Glimcher PW (1999): Neural correlates of decision variables in parietal cortex. Nature 400:233-238.

42. Paulus MP, Rogalsky C, Simmons A, Feinstein JS, Stein MB (2003): Increased activation in the right insula during risk-taking decision making is related to harm avoidance and neuroticism. Neuroimage 19:14391448.

43. Kuhnen CM, Knutson $B$ (2005): The neural basis of financial risk taking. Neuron 47:763-770.

44. O'Connor J, Dickerson M (2003): Impaired control over gambling in gaming machine and off-course gamblers. Addiction 98:53-60.

45. Kerns JG, Cohen JD, MacDonald AW 3rd, Cho RY, Stenger VA, Carter CS (2004): Anterior cingulate conflict monitoring and adjustments in control. Science 303:1023-1026.

46. Carter CS, Macdonald AM, Botvinick M, Ross LL, Stenger VA, Noll D, et al. (2000): Parsing executive processes: Strategic vs. evaluative functions of the anterior cingulate cortex. Proc Natl Acad Sci U S A 97:1944-1948.

47. Rushworth MFS, Walton ME, Kennerley SW, Bannerman DM (2004): Action sets and decisions in the medial frontal cortex. Trends Cogn Sci 8:410-417.

48. Huettel SA, Stowe CJ, Gordon EM, Warner BT, Platt ML (2006): Neural signatures of economic preferences for risk and ambiguity. Neuron 49: 765-775.

49. Gusnard DA, AkbudakE, Shulman GL, Raichle ME (2001): Medial prefrontal cortex and self-referential mental activity: Relation to a default mode of brain function. Proc Natl Acad Sci U S A 98:4259-4264.

50. O'Doherty J, Dayan P, Schultz J, Deichmann R, Friston K, Dolan RJ (2004): Dissociable roles of ventral and dorsal striatum in instrumental conditioning 10.1126/science.1094285. Science 304:452-454.

51. Samejima K, Ueda Y, Doya K, Kimura M (2005): Representation of actionspecific reward values in the striatum. Science 310:1337-1340.

52. Bush G, Vogt BA, Holmes J, Dale AM, Greve D, Jenike MA, et al. (2002): Dorsal anterior cingulate cortex: A role in reward-based decision making. Proc Natl Acad Sci U S A 99:523-528.

53. Kennerley SW, Walton ME, Behrens TE, Buckley MJ, Rushworth MF (2006): Optimal decision making and the anterior cingulate cortex. Nat Neurosci 9:940-947. 